\title{
Design Based Routing with Waveband and Wavelength Assignment in Wavelength Division Multiplexing Networks using Multi-Granular Optical Cross-Connects
}

\author{
Akhtar Nawaz Khan and Poompat Saengudomlert \\ Telecommunications Field of Study, School of Engineering and Technology, \\ Asian Institute of Technology, Thailand \\ Email: \{akhtar.nawaz.khan, poompats\}@ait.ac.th
}

\begin{abstract}
In this paper, we extend design based routing (DBR), which is originally proposed for opaque wavelength division multiplexing (WDM) networks without waveband switching (WBS), to transparent WDM networks with WBS. We formulate and solve the corresponding integer linear programming (ILP) problems to facilitate the use of DBR for transparent WDM networks with WBS, referred to as DBR with Waveband and Wavelength Assignment (DBRWWA). The goal of DBRWWA is to minimize the total link cost subject to the constraints on traffic demands and on network resources. DBRWWA guides online routing of dynamic lightpath requests in WDM networks with WBS, where traffic engineered routes are computed offline based on the prior information on traffic demand statistics. We investigate two novel heuristics, called the maximum waveband-wavelength first (MaxWWF) algorithm and the minimum waveband-wavelength first (MinWWF) algorithm, as well as existing first-fit and random-fit algorithms. The proposed heuristics consider all properties of WBS, including waveband and wavelength continuity constraints. We consider end-to-end WBS (ETE-WBS) for lightpath grouping. The online heuristics are combined with shortest path first (SPF) routing, DBR, and DBRWWA to support dynamic traffic. Simulation results show that DBRWWA has on average lower blocking probability compared to DBR, while both DBRWWA and DBR greatly outperform SPF routing in terms of the blocking probability.
\end{abstract}

Index Terms-designed based routing, multi-granular optical cross-connect, transparent networks, waveband switching, wavelength division multiplexing.

\section{INTRODUCTION}

Waveband switching (WBS) is a promising candidate to overcome the limitations faced in switching wavelength channels in optical networks based on wavelength division multiplexing (WDM). In WDM, the bandwidth of an optical fiber is divided into a large number of channels each of which is called a wavelength. In a WDM network, a switching port is required for a ligthpath request to enter an input or to leave an output of a switching node called an optical cross-connect (OXC). An OXC-based switching node may have several input fibers each consisting of hundreds of wavelengths [1]. Such a large number of input lightpaths has a direct impact on the

Manuscript received March 03, 2012; revised June 02, 2012; accepted June 13,2012 . associated sizes, complexity in control and management, and costs of OXCs [2].

In order to control the associated limitations faced in switching wavelength channels, which is referred to as wavelength switching (WLS), the concept of WBS has been introduced in [2], [3]. In WBS, several wavelengths are grouped into a single unit called a waveband (or simply a band). Similarly, several bands are grouped into a fiber. The number of wavelengths in a band is called the waveband granularity. A fiber or a band can be switched as a single entity at a switching node using common switch ports at the input and the output. An OXC capable of both wavelength switching and waveband switching is called a multi-granular optical cross-connect (MG-OXC).

Different MG-OXC architectures have been studied in [2][8]. An MG-OXC-based switching node architecture that is dynamic, flexible, and efficient is described in [6], [10], [11]. The most general MG-OXC-based switching node architecture has three layers consisting of the fiber cross-connect (FXC) layer, the band cross-connect (BXC) layer, and the wavelength cross-connect (WXC) layer. The architecture is dynamic and flexible in nature as any fiber can be demultiplexed into bands and any band can be demultiplexed into wavelengths.

Different lightpath grouping schemes have been developed and their performances in WDM networks with WBS have been investigated in [2], [6], [12], [13]. In general, lightpath grouping strategies can be divided into end-to-end WBS (ETE-WBS), same-destination intermediate WBS (SDIT-WBS), same-source intermediate WBS (SS-IT-WBS), and sub-path WBS grouping. Lightpath grouping affects the blocking probability of a lightpath request and the sizes of MGOXCs. It has been demonstrated that SD-IT-WBS can reduce the size of MG-OXCs by a factor of 2 or more [2], [12]. Similarly, ETE-WBS can also reduce the size of MG-OXCs while outperforming SD-IT-WBS in terms of the blocking probability [12].

In WDM networks with WLS, the problem of setting up lightpaths from source to destination nodes for traffic arrivals is called the routing and wavelength assignment (RWA) problem [14]. WDM networks in which a transmitted data signal remains in the optical domain from its transmitter at the source node to its receiver at the destination node are 
called transparent networks. The corresponding condition that the signal must be on the same wavelength on all links on its path is called the wavelength continuity constraint [14], [15]. In WDM networks with WBS, the allocation of a waveband to a lightpath is also considered along with RWA.

The RWA problem is divided into static and dynamic cases, depending on the types of lightpath requests [14], [16]. The static RWA problem can be formulated as an ILP problem which is known to be NP-complete [17]. The ILP formulations for RWA problems in WDM networks with only WLS cannot be applied directly to MG-OXC-based networks. WBS requires further constraints besides the wavelength continuity constraint. These constraints involve waveband routing, waveband assignment, waveband grouping, waveband disaggregation, and the waveband continuity constraint [13].

Dynamic routing based on optimization with the lightpath blocking probability as a metric has been proposed for opaque WDM networks in [18], and is called design based routing (DBR). DBR utilizes optimization as well as the current network state. The goal of optimization in DBR is to minimize the total link cost in the network subject to the constraints on traffic demands. DBR guides online routing of dynamic lightpath requests between source nodes and destination nodes, where optimized paths are computed offline based on prior information on traffic demands. However, in [18], an ILP problem is formulated for DBR in opaque networks with WLS but no WBS.

An ILP formulation for a MG-OXC-based network is proposed in [5] with the objective to minimize the number of switch ports at the WXC layer and the BXC layer subject to minimum link loading. However, wavelength converters are used at the WXC layer. Therefore, wavelength continuity is not considered in the ILP formulation in [5]. Similarly, constraint programming for WDM networks with WBS is considered in [7] with the objective to minimize the switch capacity at the FXC layer as well as the BXC layer. In [19], an ILP problem is formulated to maximize the number of supported lightpath requests in a WDM network with WBS. However, wavelength conversion is assumed. Over all, all these ILP formulations for WDM networks with WBS cannot be directly applied to routing of dynamic lightpath requests.

For dynamic traffic, heuristic methods are generally followed for the waveband and wavelength assignment in WDM networks with WBS. Several heuristics have been proposed including the maximum overlap ratio (MOR) algorithm, the maximum interference length in band (MILB) algorithm, firstfit, random-fit, and integrated intermediate waveband switching (IIWBS) [9]-[13]. These heuristics utilize k-shortest paths between source and destination nodes, which are computed offline, and do not utilize traffic information. Therefore, they may overutilize some links while underutilizing others.

In this work, we modify DBR for dynamic routing of lightpath requests to be applicable in transparent WDM networks with WBS. The overall contributions in this paper can be summarized as follows.

- We modify DBR, which was originally proposed in [18] for opaque networks, to be applicable for transparent optical networks with WBS. The resultant scheme is called DBR with waveband and wavelength assignment (DBRWWA), and is used for online routing of dynamic lightpath requests. The wavelength and waveband continuity constraints are considered in the ILP formulation for DBRWWA, which overcomes the limitations faced by other ILP formulations in [5], [7], [18], [19]. We solve the corresponding ILP problem to facilitate the use of DBRWWA for routing of dynamic ligthpath requests.

- For waveband and wavelength assignment, we investigate two novel heuristics, called the maximum wavebandwavelength first (MaxWWF) algorithm and the minimum waveband-wavelength first (MinWWF) algorithm. These heuristics consider all properties of WBS including the waveband and wavelength continuity constraints, waveband assignment, waveband grouping at source nodes, waveband disaggregation at destination nodes, and wavelength assignment within each waveband. For comparison, we also consider first-fit and random-fit heuristics. These heuristics are combined with shortest path first (SPF) routing, DBR, and DBRWWA to support dynamic traffic.

- We demonstrate through computer simulations that the proposed DBRWWA scheme provides on average a lower blocking probability of a lightpath request when compared to DBR and SPF.

The paper is organized as follows. Section II consists of background on DBR. Section III describes a two-layer MGOXC-based switching node architecture, ILP for DBRWWA, and the proposed heuristics. Section IV presents the overall DBRWWA algorithm for WDM transparent networks with WBS. In Section V, the performances of DBRWWA and existing routing heuristics are investigated through simulation results in different scenarios. Finally, Section VI concludes the paper.

\section{BACKGROUND ON DBR}

A WDM network consists of OXCs that have the ability to transmit, receive, and route lightpath requests from inputs to outputs. In an opaque WDM network, any wavelength can be used to carry a lightpath request on any link along the path from the source node to the destination node [20], [21]. An opaque WDM network consists of opaque switching nodes whose architecture is shown in Fig. 1 [15], [22].

DBR is formulated in [18] as an optimization problem for opaque WDM networks which does not consider the wavelength continuity constraint. The optimization objective is to minimize the total link cost subject to the constraints on traffic demands. In DBR, traffic engineering paths are computed offline based on prior information in traffic demand statistics, and are utilized for online routing of dynamic lightpath requests in opaque WDM networks.

The parameters, variables, and constraints used in the DBR optimization model for opaque WDM networks without WBS are listed in Table I.

Objective: The objective of DBR is to minimize the total link cost in a WDM opaque network as shown in Eq.(1). 


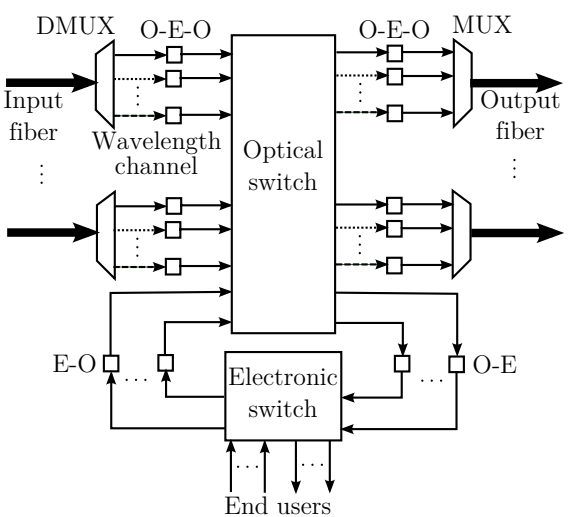

E-O: electronic-to-optical converter O-E: optical-to-electronic converter O-E-O: optical-to-electronic-to-optical converter MUX/DMUX: multiplexer/demultiplexer

Figure 1. Generic opaque optical switching node architecture (adapted from [15], [22])

TABLE I.

INPUT AND OUTPUT PARAMETERS IN ILP FOR DBR

\begin{tabular}{|l|l|}
\hline \multicolumn{2}{|c|}{ INPUT PARAMETERS } \\
\hline \hline \multicolumn{2}{|c|}{ Network parameters } \\
\hline $\mathcal{W}$ & set of wavelength channels in each fiber, indexed from 1 to $W$ \\
\hline $\mathcal{S}$ & $\begin{array}{l}\text { set of directed links, where each directed link } l \in \mathcal{L} \text { is a single } \\
\text { fiber for transmission in one direction }\end{array}$ \\
\hline$w_{l}$ & set of source-destination (s-d) pairs with nonzero traffic \\
\hline \multicolumn{2}{|c|}{ Traffic parameters } \\
\hline$\alpha^{s}$ & $\begin{array}{l}\text { lightpath arrival rate for s-d pair } s, \text { where each lightpath takes } \\
\text { up a full wavelength }\end{array}$ \\
\hline$\gamma$ & target upper bound on lightpath blocking probability \\
\hline \multicolumn{2}{|c|}{ Oameters } \\
\hline $\mathcal{P}$ & set of paths for all s-d pairs \\
\hline $\mathcal{P}^{s}$ & set of paths for s-d pair $s$ \\
\hline $\mathcal{P}_{l}$ & set of paths that use link $l$ \\
\hline \hline \multicolumn{2}{|c|}{ OUTPU PARAMETERS (VARIABLES) } \\
\hline \hline$x^{p}$ & capacity assigned (in wavelengths) on path $p$ according to DBR \\
\hline$\lambda^{s}$ & total assigned capacity for s-d pair $s$ \\
\hline$\lambda_{l}$ & total assigned capacity on link $l$ \\
\hline
\end{tabular}

$$
\operatorname{minimize} \sum_{l \in \mathcal{L}} w_{l} \lambda_{l}
$$

\section{Constraints:}

The total assigned capacity on each link $l$ is equal to the total assignment on the paths that use the link, as shown in Eq.(2).

$$
\forall l \in \mathcal{L}, \quad \sum_{p \in \mathcal{P}_{l}} x^{p}=\lambda_{l}
$$

The link capacity limitation is given in Eq.(3).

$$
\forall l \in \mathcal{L}, \quad \sum_{p \in \mathcal{P}_{l}} x^{p} \leq W
$$

The total capacity assigned for each s-d pair $s$ is equal to the total assignment on the paths for the s-d pair, as shown in Eq.(4).

$$
\forall s \in \mathcal{S}, \quad \sum_{p \in \mathcal{P} s} x^{p}=\lambda^{s}
$$

The bound on the blocking probability is given in Eq.(5)

$$
\forall s \in \mathcal{S}, \quad \operatorname{Erl}\left(\alpha^{s}, \lambda^{s}\right) \leq \gamma
$$

where $\operatorname{Erl}\left(\alpha^{s}, \lambda^{s}\right)$ is the Erlang B formula given in Eq.(6).

$$
\operatorname{Erl}\left(\alpha^{s}, \lambda^{s}\right)=\frac{\frac{\left(\alpha^{s}\right)^{\lambda^{s}}}{\lambda^{s !}}}{\sum_{i=0}^{\lambda^{s}} \frac{\left(\alpha^{s}\right)^{i}}{i !}}
$$

The integer constraints are given in Eq.(7) and Eq.(8).

$$
\begin{gathered}
\forall p \in \mathcal{P}, \quad x^{p} \in\{0,1, \cdots, W\} \\
\forall s \in \mathcal{S}, \quad \lambda^{s} \in \mathbb{Z}^{+}
\end{gathered}
$$

The nonlinear constraints in Eq.(5) make the problem nonlinear. Therefore, each nonlinear constraint is replaced by computing the minimum value of $\lambda^{s}$, denoted by $\tilde{\lambda}^{s}$, as given in Eq.(9).

$$
\tilde{\lambda}^{s}=\arg \min _{\lambda \in\{1,2,3, \ldots\}} \operatorname{Erl}\left(\alpha^{s}, \lambda\right) \leq \gamma
$$

The value $\tilde{\lambda}^{s}$ is then used as a parameter in the optimization formulation instead of $\lambda^{s}$, while all nonlinear constraints are dropped. In addition, the constraints in Eq.(2) can be absorbed into the objective function. The objective function in Eq.(1) can then be rewritten as in Eq.(10), which is shown below together with all the constraints.

\section{Objective :}

$$
\operatorname{minimize} \sum_{l \in \mathcal{L}} w_{l}\left(\sum_{p \in \mathcal{P}_{l}} x^{p}\right)
$$

\section{Constraints:}

$$
\begin{gathered}
\forall l \in \mathcal{L}, \quad \sum_{p \in \mathcal{P}_{l}} x^{p}=W \\
\forall s \in \mathcal{S}, \quad \sum_{p \in \mathcal{P}^{s}} x^{p}=\tilde{\lambda}^{s} \\
\forall p \in \mathcal{P}, \quad x^{p} \in\{0,1 . \cdots, W\}
\end{gathered}
$$

\section{PROBLEM Formulation}

\section{A. MG-OXC-Based Switching Node Architecture}

The most general MG-OXC-based switching node architecture has three layers consisting of the FXC layer, the BXC layer, and the WXC layer [11]. We consider a WDM network in which each link consists of two fibers, one for the transmission in each direction. We assume that each switching node has two layers; the FXC layer is removed for simplicity. A two-layer MG-OXC-based switching node consists of the WXC layer and the BXC layer, as shown in Fig. 2, and has the same working principle as given in [11]. The node architecture in Fig. 2 is dynamic; any band can be demultiplexed into wavelengths. Also, the WXC layer interfaces with access networks, i.e., end users.

The BXC layer consists of multiple optical switching fabrics, one for each waveband. Similarly, the WXC layer consists of multiple optical switching fabrics, one for each wavelength, as shown in Fig. 2. There is no optical-to-electronic-to-optical (O-E-O) converter at the input and the output in the WXC 


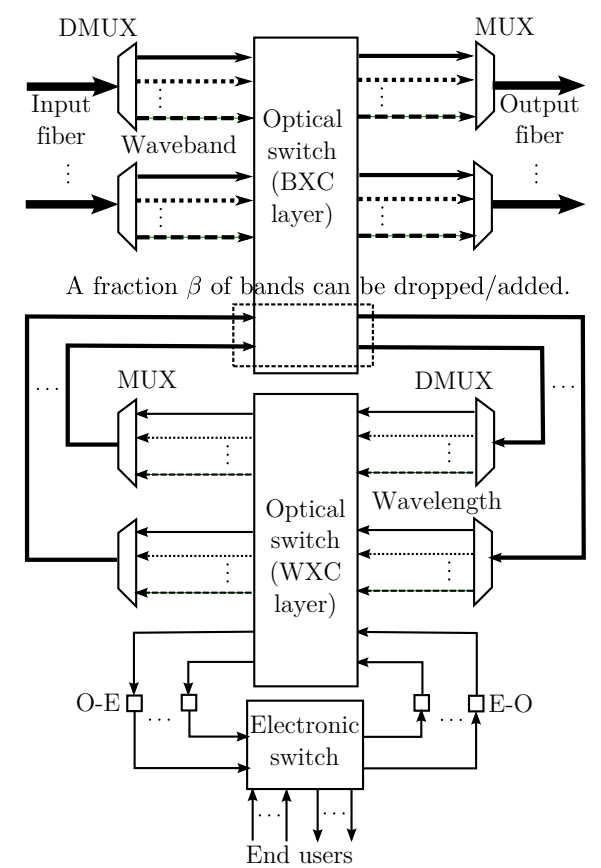

Figure 2. Two-layer MG-OXC-based switching node architecture consisting of the WXC layer and the BXC layer (adapted from [11])

layer as well as the BXC layer. Therefore, both the wavelength and waveband continuity constraints are considered. End users' lightpaths enter and leave the network through electronic switch ports and through electronic-to-optical (E$\mathrm{O})$ and optical-to-electronic (O-E) converters respectively.

In Fig. $2, \beta \leq 1$ is the switch parameter defined as the ratio of the number of bands to be demultiplexed into wavelengths (from the BXC layer to the WXC layer) to the total number of bands available at the input of the BXC layer. If $\beta=1$, all bands can be demultiplexed into wavelengths and a MG-OXCbased switching node will function like an ordinary OXCbased switching node. In addition, let $G$ denote the granularity of each waveband.

\section{B. ILP Formulation for DBRWWA}

DBRWWA is formulated as an optimization problem for WDM networks with WBS. The optimization objective is to minimize the total link cost subject to the constraints on traffic demands. In DBRWWA, traffic engineering paths are computed offline based on prior information on traffic demand statistics using k-shortest paths. The traffic engineering paths are then utilized for online routing of dynamic lightpath requests in WDM networks with WBS. Moreover, DBRWWA considers the waveband and wavelength continuity constraints.

The parameters, variables, and constraints used in the DBRWWA optimization model are listed in Table II.

Objective: The objective of DBRWWA is to minimize the total link cost in the network as shown in Eq.(11).

$$
\operatorname{minimize} \sum_{l \in \mathcal{L}} w_{l} \lambda_{l}
$$

TABLE II.

INPUT AND OUTPUT PARAMETERS IN ILP FOR DBRWWA

\begin{tabular}{|l|l|}
\hline \multicolumn{1}{|c|}{ INPUT PARAMETERS } \\
\hline \hline \multicolumn{2}{|c|}{ Network parameters } \\
\hline $\mathcal{W}$ & set of wavelength channels in each fiber, indexed from 1 to $W$ \\
\hline $\mathcal{B}$ & set of wavebands in each fiber, indexed from 1 to $B$ \\
\hline $\mathcal{W}_{b}$ & set of wavelengths in waveband $b$ \\
\hline $\mathcal{L}$ & $\begin{array}{l}\text { set of directed links, where each directed link } l \in \mathcal{L} \text { is a single } \\
\text { fiber for transmission in one direction }\end{array}$ \\
\hline $\mathcal{N}$ & set of nodes \\
\hline $\mathcal{S}$ & set of source-destination (s-d) pairs with nonzero traffic \\
\hline$w_{l}$ & cost of using one wavelength channel on link $l$ \\
\hline$G$ & waveband granularity (number of wavelengths in each waveband) \\
\hline$\Delta_{n}$ & limit on input/output ports in the WXC layer at node $n$ \\
\hline \multicolumn{2}{|c|}{ Traffic parameters } \\
\hline$\alpha^{s}$ & $\begin{array}{l}\text { lightpath arrival rate for s-d pair } s, \text { where each lightpath takes } \\
\text { up a full wavelength }\end{array}$ \\
\hline$\gamma$ & target upper bound on lightpath blocking probability \\
\hline \multicolumn{2}{|c|}{ Path parameters } \\
\hline $\mathcal{P}$ & set of paths for all s-d pairs \\
\hline $\mathcal{P}^{s}$ & set of paths for s-d pair $s$ \\
\hline $\mathcal{P}_{l}$ & set of paths that use link $l$ \\
\hline $\mathcal{P}_{n}^{S}$ & set of paths with node $n$ as the source node \\
\hline $\mathcal{P}_{n}^{D}$ & set of paths with node $n$ as the destination node \\
\hline $\mathcal{P}_{n}^{I}$ & set of paths with node $n$ as an intermediate node \\
\hline \hline \multicolumn{2}{|c|}{ OUTPUT PARAMETERS (VARIABLES) } \\
\hline \hline$x_{w}^{P}$ & $\begin{array}{l}\text { capacity assigned (in wavelengths) on path } p \text { on wavelength } w \\
\text { according to DBR }\end{array}$ \\
\hline$y_{b}^{p}$ & capacity assigned (in wavebands) on path $p$ on waveband $b$ \\
\hline$\lambda^{s}$ & total assigned capacity for s-d pair $s$ \\
\hline$\lambda_{l}$ & total assigned capacity on link $l$ \\
\hline &
\end{tabular}

\section{Constraints:}

The total assigned capacity on each link $l$ is equal to the total assignment on the paths that use the link, as shown in Eq.(12).

$$
\forall l \in \mathcal{L}, \quad \sum_{p \in \mathcal{P}_{l}, w \in \mathcal{W}} x_{w}^{p}=\lambda_{l}
$$

The link capacity limitation and the wavelength collision constraint on each link is given in Eq.(13).

$$
\forall l \in \mathcal{L}, \forall w \in \mathcal{W}, \quad \sum_{p \in \mathcal{P}_{l}} x_{w}^{p} \leq 1
$$

The total capacity assigned for s-d pair $s$ must be at least the required capacity based on the blocking probability, as shown in Eq.(14).

$$
\forall s \in \mathcal{S}, \quad \sum_{p \in \mathcal{P}^{s}, w \in \mathcal{W}} x_{w}^{p} \geq \lambda^{s}
$$

The bound on the blocking probability for each s-d pair $s$ is expressed in Eq.(15).

$$
\forall s \in \mathcal{S}, \quad \operatorname{Erl}\left(\alpha^{s}, \lambda^{s}\right) \leq \gamma
$$

The constraints for wavebanding are given in Eq.(16). In particular, a wavelength can only be used if its waveband is used.

$$
\forall p \in \mathcal{P}, \forall b \in \mathcal{B}, \forall w \in \mathcal{W}_{b}, \quad x_{w}^{p} \leq y_{b}^{p}
$$

The number of wavelength channels into/out of node $n$ is given in Eq.(17).

$$
\sum_{w \in \mathcal{W}} \sum_{p \in \mathcal{P}_{n}^{S} \cup \mathcal{P}_{n}^{D} \cup \mathcal{P}_{n}^{I}} x_{w}^{p}
$$


The reduction of WXC ports due to WBS at the input/output of node $n$ is given in Eq.(18). Note that this reduction is due to the bypass traffic. For each waveband that bypasses the WXC layer, $G$ WXC input/output ports can be reduced at the intermediate node $n$.

$$
\sum_{b \in \mathcal{B}} \sum_{p \in \mathcal{P}_{n}^{I}} G y_{b}^{p}
$$

The number of WXC ports used at the input/output of node $n$ cannot exceed the available number of ports, as shown in Eq.(19).

$$
\forall n \in \mathcal{N}, \quad \sum_{w \in \mathcal{W}_{p} \in \mathcal{P}_{n}^{S} \cup \mathcal{P}_{n}^{D} \cup \mathcal{P}_{n}^{I}} x_{w}^{p}-\sum_{b \in \mathcal{B}_{p} \in \mathcal{P}_{n}^{I}} G y_{b}^{p} \leq \Delta_{n}
$$

The integer constraints are given in Eq.(20) - Eq.(22).

$$
\begin{gathered}
\forall p \in \mathcal{P}, w \in \mathcal{W}, \quad x_{w}^{p} \in\{0,1\} \\
\forall p \in \mathcal{P}, b \in \mathcal{B}, \quad y_{b}^{p} \in\{0,1\} \\
\forall s \in \mathcal{S}, \quad \lambda^{s} \in \mathbb{Z}^{+}
\end{gathered}
$$

The nonlinear constraints in Eq.(15) make the problem nonlinear. Therefore, the nonlinear constraints are replaced by computing the minimum value of $\lambda^{s}$, denoted by $\tilde{\lambda}^{s}$, which is given in Eq.(9). The value of $\tilde{\lambda}^{s}$ is then used as a parameter in the optimization formulation instead of $\lambda^{s}$.

The objective function in Eq.(11) can be rewritten as given in Eq.(23) together with all the constraints below.

Objective:

$$
\operatorname{minimize} \sum_{l \in \mathcal{L}} w_{l}\left(\sum_{p \in \mathcal{P}_{l}, w \in \mathcal{W}} x_{w}^{p}\right)
$$

\section{Constraints:}

$$
\begin{gathered}
\forall l \in \mathcal{L}, \forall w \in \mathcal{W}, \quad \sum_{p \in \mathcal{P}_{l}} x_{w}^{p} \leq 1 \\
\forall s \in \mathcal{S}, \sum_{p \in \mathcal{P}^{s}, w \in \mathcal{W}} x_{w}^{p} \geq \tilde{\lambda}^{s} \\
\forall p \in \mathcal{P}, \forall b \in \mathcal{B}, \forall w \in \mathcal{W}_{b}, \quad x_{w}^{p} \leq y_{b}^{p} \\
\forall n \in \mathcal{N}, \quad \sum_{w \in \mathcal{W}} \sum_{p \in \mathcal{P}_{n}^{S} \cup \mathcal{P}_{n}^{D} \cup \mathcal{P}_{n}^{I}} x_{w}^{p}-\sum_{b \in \mathcal{B}} \sum_{p \in \mathcal{P}_{n}^{I}} G y_{b}^{p} \leq \Delta_{n} \\
\forall p \in \mathcal{P}, w \in \mathcal{W}, x_{w}^{p} \in\{0,1\} \\
\forall p \in \mathcal{P}, b \in \mathcal{B}, y_{b}^{p} \in\{0,1\}
\end{gathered}
$$

To indicate the computational complexity in solving the ILP problem for DBRWWA, Table III provides the number of decision variables and the number of constraints (not including integer constraints) in terms of the network and traffic parameters.
TABLE III

NUMBER OF VARIABLES AND NUMBER OF CONSTRAINTS IN ILP FOR DBRWWA

\begin{tabular}{|l|c|}
\hline Type & Total number \\
\hline \hline Variables & $|\mathcal{P}||\mathcal{W}|+|\mathcal{P}||\mathcal{B}|$ \\
\hline Constraints & $|\mathcal{L}||\mathcal{W}|+|\mathcal{S}|+|\mathcal{P}||\mathcal{W}|+|\mathcal{N}|$ \\
\hline
\end{tabular}

\section{Online Heuristics for Waveband and Wavelength Assign- ment}

We now describe the heuristics that we consider for waveband and wavelength assignment. The waveband and wavelength continuity constraints are taken into account in the heuristics. The ETE-WBS scheme is used for lightpath grouping. These heuristics can be implemented as online algorithms, and are combined with routing based on SPF, DBR, and DBRWWA to support dynamic traffic.

1) First-Fit and Random-Fit Algorithms: First-fit and random-fit algorithms are considered in [9]-[11]. However, in previous works, both algorithms employ k-shortest path routing for a lightpath request. The first-fit algorithm selects the first available waveband along the selected path from the source node to the destination node, and assigns the first available wavelength in the waveband. However, the randomfit algorithm randomly selects a waveband from the set of available wavebands along the selected path, and randomly assigns an available wavelength in the waveband. In both cases, if a wavelength is not available in any existing waveband, a new waveband is created. If a new waveband cannot be created, a lightpath request is blocked.

2) MaxWWF Algorithm: We investigate the maximum waveband-wavelength first (MaxWWF) assignment heuristic, which is based on the most-used wavelength assignment reported in [14], [23]. In [14], it has been shown through simulation results that the most-used wavelength assignment slightly outperforms the first-fit wavelength assignment.

In the MaxWWF assignment heuristic, the most frequently used waveband along the path is assigned to a ligthpath request. In the case of a tie between two or more available wavebands, the waveband with the minimum index is selected.

Similarly, the most-used wavelength within the selected waveband is assigned to a lightpath request. In case of a tie, the wavelength with the minimum index is selected. If a wavelength is not available in any existing waveband, a new waveband is created. If a new waveband cannot be created, a lightpath request is blocked. Note that the MaxWWF algorithm requires information regarding the wavebands and the wavelengths within each waveband that are most frequently used.

3) MinWWF Algorithm: We also investigate the minimum waveband-wavelength first (MinWWF) assignment heuristic, which is based on the least-used wavelength assignment reported in [14], [23]. In the MinWWF assignment heuristic, the least-used waveband along the path is assigned to a ligthpath request. In the case of a tie, the waveband with the minimum index is selected. Similarly, the least-used wavelength within the selected waveband is assigned to a lightpath request. In case of a tie, the wavelength with the minimum index is 
selected.

If a wavelength is not available in any existing waveband, a new waveband is created. If a new waveband cannot be created, a lightpath request is blocked. The MinWWF assignment heuristic tries to balance the loads among all wavebands and among all wavelengths within wavebands. Similar to MaxWWF, the MinWWF heuristic requires information regarding the wavebands and the wavelengths within each waveband that are least frequently used.

\section{Dynamic Routing, WaVeband And WAVELEngth ASSIGNMENT ALGORITHMS}

\section{A. $S P F+$ Online Heuristics}

In this algorithm, SPF routing is considered for the routing subproblem, while online heuristics consisting of first-fit, random-fit, MaxWWF, and MinWWF algorithms are used for waveband and wavelength assignment subproblems. SPF routing tries to support online routing of dynamic ligthpath requests by utilizing shortest paths from source nodes to destination nodes. However, these shortest paths are computed offline and do not consider the current network loads.

\section{B. $D B R+$ Online Heuristics}

In this algorithm, DBR is considered for the routing subproblem for dynamic lightpath requests. DBR effectively guides online routing of dynamic lightpath requests between source nodes and destination nodes, where optimized paths are computed offline. DBR tries to support a lightpath request by utilizing a traffic engineering path previously found. If an optimized path from the source node to the destination node is not available, a ligthpath request is blocked.

\section{DBRWWA + Online Heuristics}

In this algorithm, DBRWWA is considered for the routing subproblem. DBRWWA also tries to support a lightpath request by utilizing a traffic engineering path previously found. However, DBRWWA tries to follow different optimized paths as compared to DBR, where the latter does not consider the wavelength and waveband continuity constraints as well as the limit on the input/output ports in the WXC layer at each node. Similarly, if an optimized path from the source node to the destination node is not available, a ligthpath request is blocked.

\section{Performance Investigation}

\section{A. Effects of Waveband Granularity}

We consider a unidirectional ring network in Fig. 3 to investigate the effects of the waveband granularity in a WDM network with WBS. The network in Fig. 3 consists of $N$ nodes. Each link is a single fiber for the transmission in one direction. Lightpath requests arrive to the network according to a Poisson process. Lightpath durations are independent and exponentially distributed with the mean of 1 time unit. The traffic load in the network is taken to be 2 Erlang, and is uniformly distributed among all s-d pairs. Each fiber consists of 6 wavelengths. SPF routing is considered for the
TABLE IV.

SIMULATION PARAMETERS FOR THE UNIDIRECTIONAL RING NETWORK

\begin{tabular}{|l|c|c|}
\hline Parameter & Notation & Value \\
\hline \hline Traffic load & $\sum_{s \in \mathcal{S}} \alpha^{s}$ & 2 Erlang \\
\hline Number of nodes & $|\mathcal{N}|$ & $\{3,4,5,10,20\}$ \\
\hline Number of lightpath requests & - & 0.1 million \\
\hline Number of wavelengths per fiber & $|\mathcal{W}|$ & 6 \\
\hline Waveband granularity & $G$ & $\{1,2,3,6\}$ \\
\hline Number of wavebands & $|\mathcal{B}|$ & $\{6,3,2,1\}$ \\
\hline
\end{tabular}

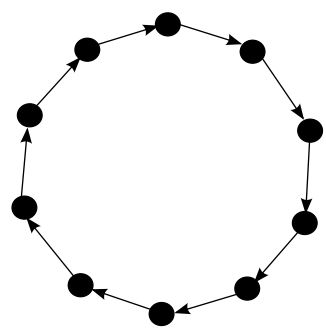

Figure 3. A unidirectional ring network.

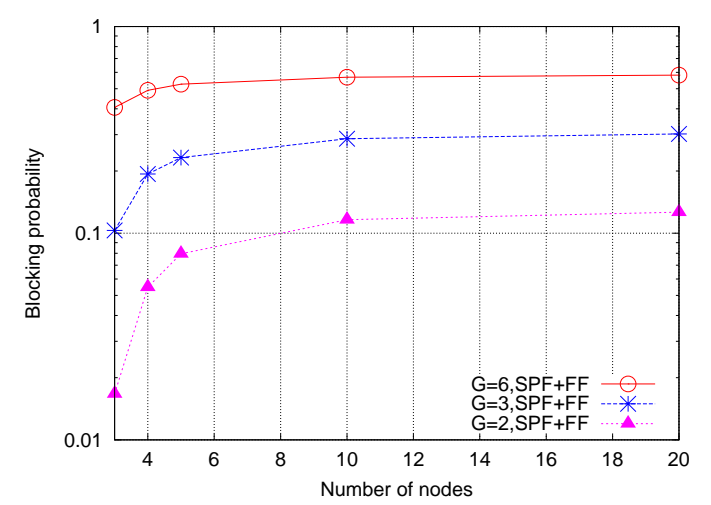

Figure 4. Blocking probability vs. number of nodes in a unidirectional ring network with different granularities

routing subproblem, while first-fit is used for the wavelength and waveband assignment subproblems. Simulation results are obtained for 0.1 million lightpath requests. Other simulation parameters are shown in Table IV. Simulation programs are developed using Python [24].

Fig. 4 and Fig. 5 show that the blocking probability increases with the granularity. In other words, the network flexibility to support dynamic traffic decreases with the granularity.

\section{B. Performance Investigation of SPF Routing + Online Heuristics}

In order to investigate the performance of the existing and the proposed online waveband and wavelength assignment heuristics, we consider the COST 239 European Optical Network (EON) topology in [25] for all simulation results. As shown in Fig. 6, the COST 239 network consists of 11 nodes and 50 directed links. Each link drawn in Fig. 6 consists of two fibers, one for the transmission in each direction. Each fiber consists of 16 wavelengths. The cost of using a wavelength channel on each link is assumed to be 1 unit. Each node is a two-layer MG-OXC-based switching node, as shown in 


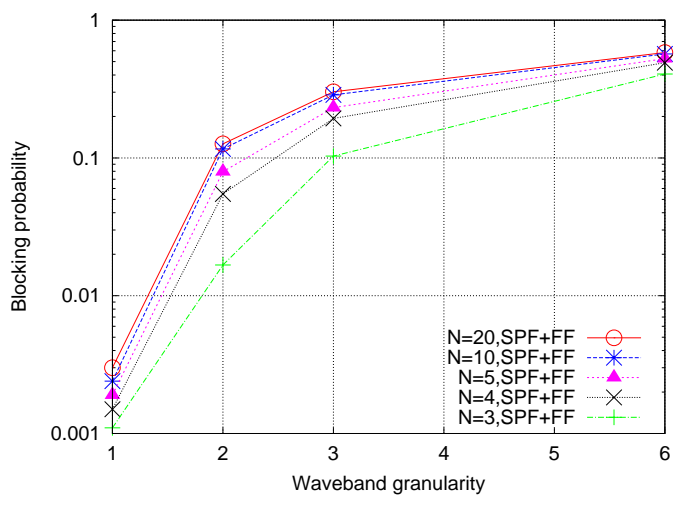

Figure 5. Effects of waveband granularity on the blocking probability with different numbers of nodes

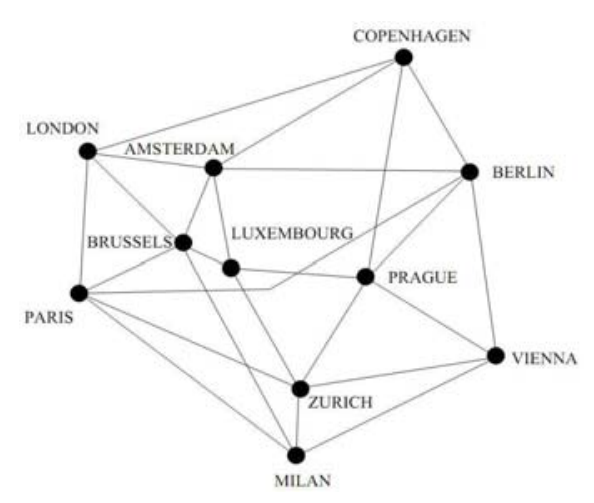

Figure 6. COST 239 network topology from [25]

Fig. 2. Lightpath requests arrive to the network according to a Poisson process. Lightpath durations are independent and exponentially distributed with the mean of 1 time unit. Traffic is considered to be symmetric and is uniformly distributed among all s-d pairs in the network. Other simulation parameters are given in Table V.

Blocking probabilities are computed for different online heuristics including first-fit, random-fit, MaxWWF, and MinWWF algorithms. The blocking probabilities are plotted against the network loads for $G \in\{1,2\}$ and $G=4$ as shown in Fig. 7 and Fig. 8 respectively. In Fig. 7 and Fig. 8, the MaxWWF algorithm has the same blocking probabilities as compared to the first-fit algorithm for waveband and wavelength assignment, while both result in lower blocking probabilities as compared to random-fit and MinWWF algorithms.

Since the first-fit and MaxWWF algorithms outperform others, we shall adopt them for waveband and wavelength assignment subproblems in the remaining of the paper.

\section{Performance Investigation of DBR + Online Heuristics}

In order to investigate the performance of DBR for WDM networks with WBS, we use the same simulation parameters as given in Table V. Moreover, lightpath requests arrive to the network according to a Poisson process. Lightpath durations are independent and exponentially distributed with the mean of 1 time unit. Traffic is considered to be symmetric and is
TABLE V.

SIMULATION PARAMETERS FOR THE COST 239 NETWORK

\begin{tabular}{|l|c|c|}
\hline Parameter & Notation & Value \\
\hline \hline Traffic load (Erlang) & $\sum_{s \in \mathcal{S}} \alpha^{S}$ & $\{140, \cdots, 220\}$ \\
\hline Number of nodes & $|\mathcal{N}|$ & 11 \\
\hline Number of directional links & $|\mathcal{L}|$ & 50 \\
\hline Number of lightpath requests & - & 1 million \\
\hline Number of s-d pairs & $|\mathcal{S}|$ & 110 \\
\hline Number of wavelengths per fiber & $|\mathcal{W}|$ & 16 \\
\hline Waveband granularity & $G$ & $\{1,2,4\}$ \\
\hline Number of wavebands & $|\mathcal{B}|$ & $\{16,8,4\}$ \\
\hline $\begin{array}{l}\text { Limit on input/out ports in the WXC } \\
\text { layer at each node } n\end{array}$ & $\Delta_{n}$ & 80 \\
\hline
\end{tabular}

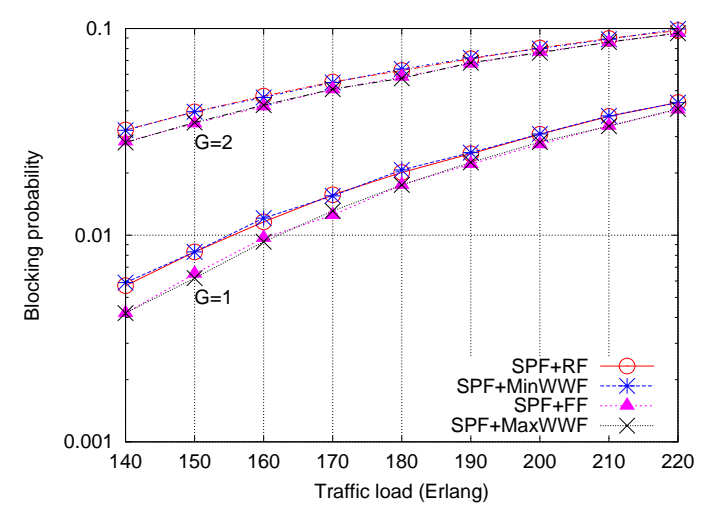

Figure 7. Blocking probability in SPF routing vs. traffic load with $G \in\{1,2\}$

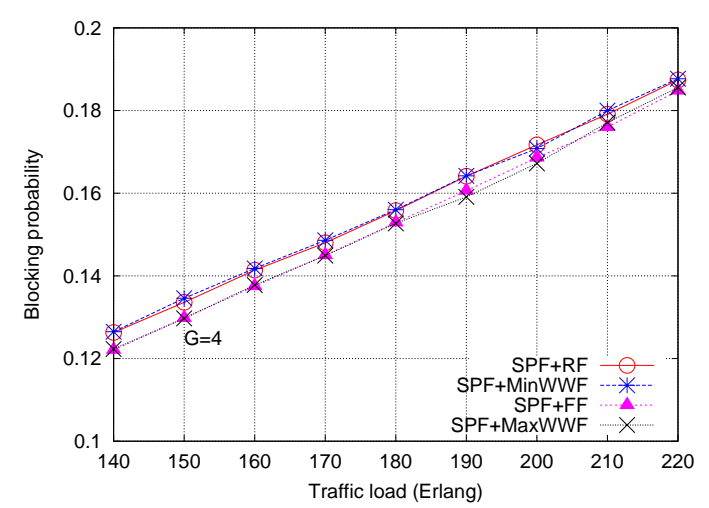

Figure 8. Blocking probability in SPF routing vs. traffic load with $G=4$

uniformly distributed among all s-d pairs. The bound on the lightpath blocking probability of DBR for WDM networks with WBS is set equal to 0.1 .

Python linear program modeler (PuLP) together with the software package GNU Linear Programming Kit (GLPK) [24] is used to solve the corresponding ILP problems to facilitate the use of DBR. The ETE-WBS scheme is used for lightpath grouping into wavebands.

Blocking probabilities are computed for different values of load and granularity as given in Table V. DBR is utilized for dynamic routing of lightpath requests while first-fit and MaxWWF algorithms are used for waveband and wavelength assignment subproblems. The blocking probabilities obtained in DBR + first-fit are compared with SPF + first-fit in 


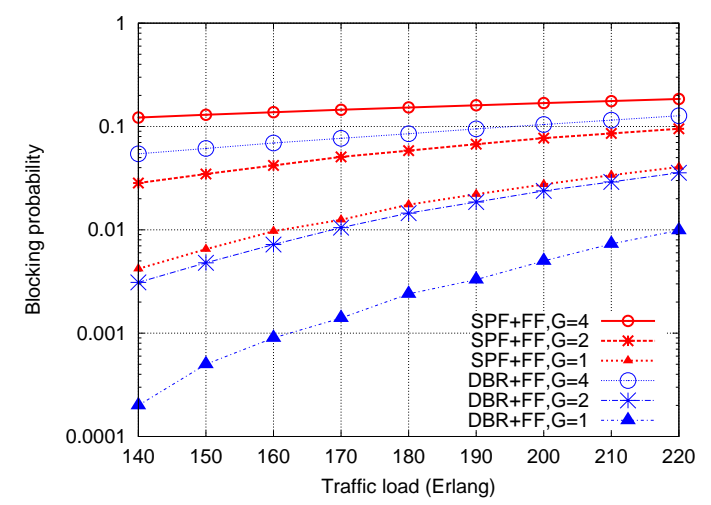

Figure 9. DBR vs. SPF routing with first-fit algorithm for waveband and wavelength assignment

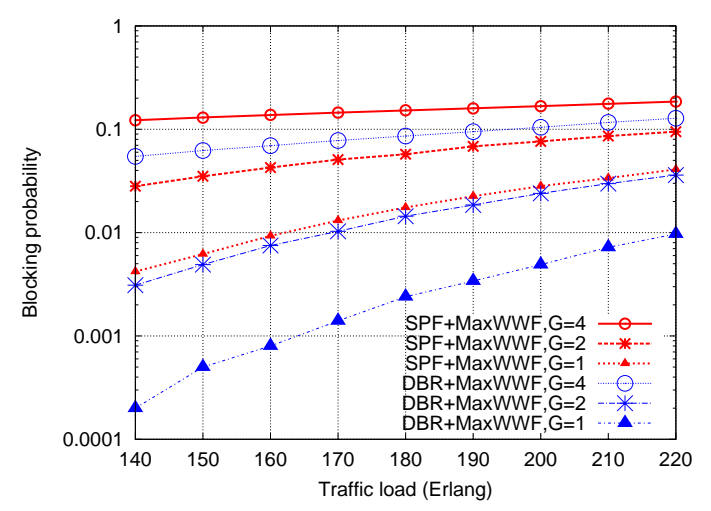

Figure 10. DBR vs. SPF routing with MaxWWF algorithm for waveband and wavelength assignment

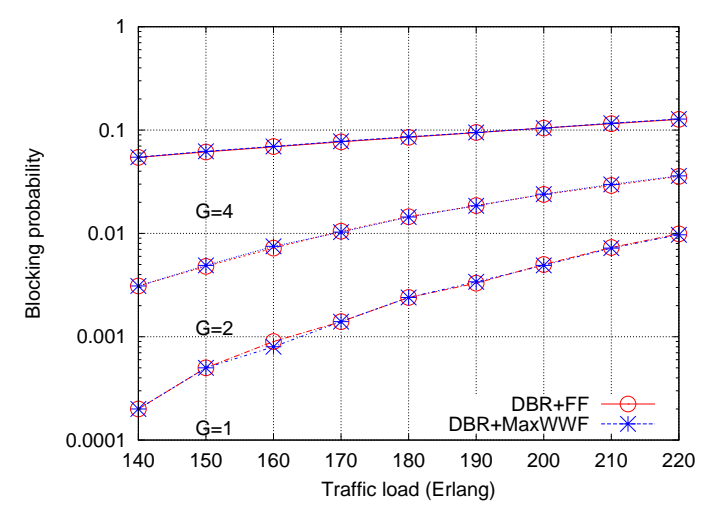

Figure 11. MaxWWF vs. first-fit using DBR for the routing subproblem for $G \in\{1,2,4\}$

Fig. 9 for $G \in\{1,2,4\}$. A similar comparison is made in Fig. 10 for $G \in\{1,2,4\}$. Fig. 9 and Fig. 10 show that DBR can effectively reduce the blocking probability in a WDM network with WBS. In addition, DBR with MaxWWF yields approximately the same blocking probability as compared to DBR with first-fit, as shown in Fig. 11.

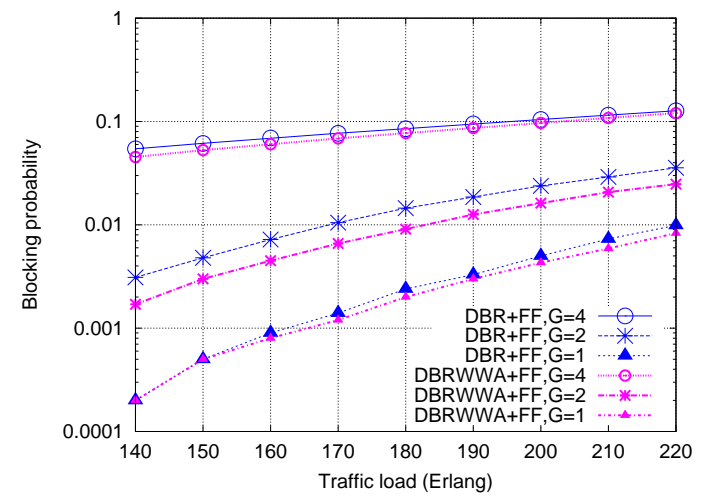

Figure 12. DBRWWA vs. DBR routing using the first-fit algorithm for waveband and wavelength assignment

\section{Performance Investigation of DBRWWA + Online Heuris- tics}

In order to investigate the performance of DBRWWA for WDM networks with WBS, we use the same simulation parameters as given in Table V. The traffic arrival process remains the same as described in the earlier sections.

Both wavelength and waveband continuity constraints are considered. The ILP problem in Section III-B is solved to facilitate the use of DBRWWA for transparent WDM networks with WBS. The ETE-WBS scheme is used for lightpath grouping. The limit on input/output ports at the WXC layer at each node is set to 80 .

DBRWWA is utilized for dynamic routing of lightpath requests while first-fit and MaxWWF algorithms are used for waveband and wavelength assignment subproblems. The blocking probabilities obtained in DBRWWA + first-fit are compared with DBR + first-fit in Fig. 12 for $G \in\{1,2,4\}$. A similar comparison is made in Fig. 13 for $G \in\{1,2,4\}$. Fig. 12 and Fig. 13 show that DBRWWA outperforms DBR in terms of the blocking probability. In addition, DBRWWA with MaxWWF yields approximately the same blocking probability as compared to DBRWWA with first-fit, as shown in Fig. 14. Fig. 15 shows the effect of $\Delta_{n}$ on the blocking probability where the network flexibility to support dynamic traffic increases with the value of $\Delta_{n}$.

\section{E. Competitive Ratios}

The performances of DBR and DBRWWA are also compared in terms of the competitive ratio adopted from [26]. The competitive ratio of DBR over SPF routing is defined as the ratio of the network throughput obtained from using DBR to the network throughput obtained from using SPF routing. Similarly, the competitive ratio of DBRWWA over SPF routing is defined as the ratio of the network throughput obtained from using DBRWWA to the network throughput obtained from using SPF routing. The network throughput is defined by the expression given in Eq.(24), where BP is the network blocking probability.

$$
\text { Throughput }=1-\mathrm{BP}
$$




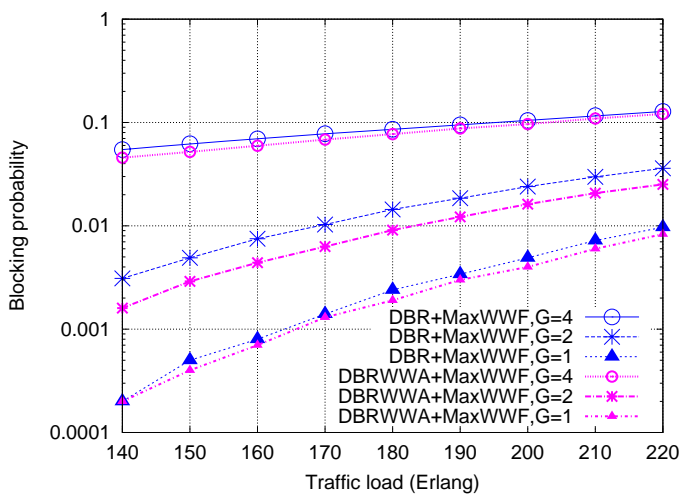

Figure 13. DBRWWA vs. DBR routing using the MaxWWF algorithm for waveband and wavelength assignment

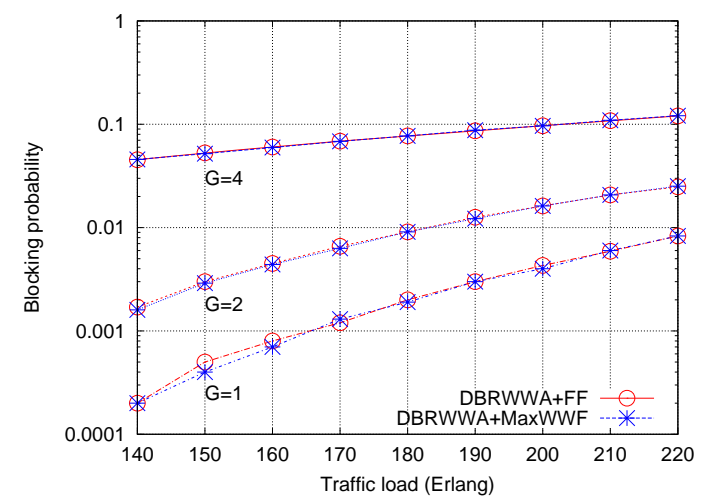

Figure 14. MaxWWF vs. first-fit using DBRWWA for the routing subproblem with $G \in\{1,2,4\}$
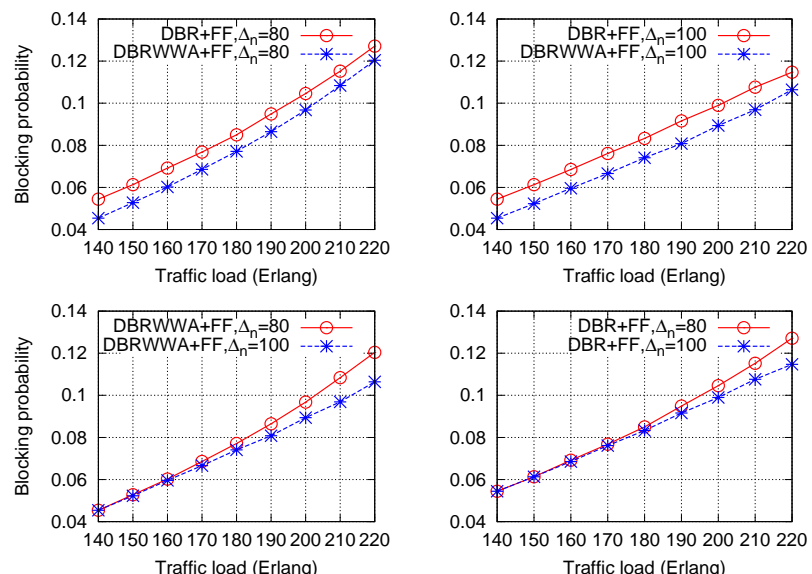

Figure 15. DBRWWA vs. DBR routing using the first-fit algorithm for waveband and wavelength assignment with $\Delta_{n}=80$ and $\Delta_{n}=100$

The competitive ratios of DBR and DBRWWA are compared for different granularities in Fig. 16 and Fig. 17, where we assume $\Delta_{n}=80$. The first-fit and MaxWWF algorithms are considered for waveband and wavelength assignment subproblems. While both DBR and DBRWWA can provide higher throughputs than SPF routing, the competitive ratios of DBRWWA are consistently higher. Finally, Fig. 18 and Fig. 19

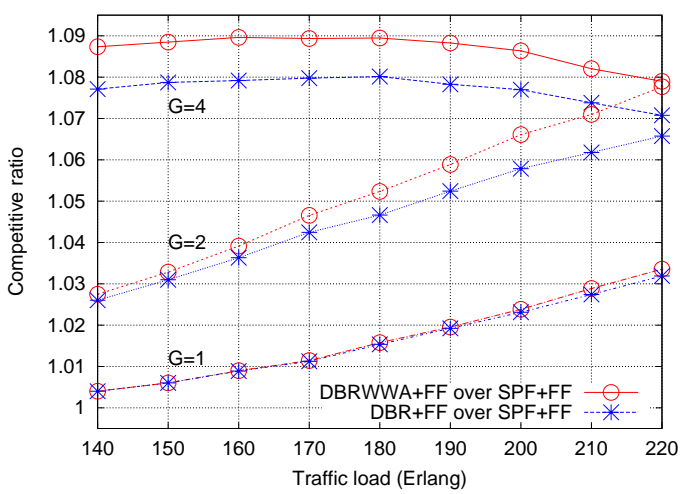

Figure 16. Competitive ratios of DBRWWA over SPF routing and of DBR over SPF routing with the first-fit algorithm

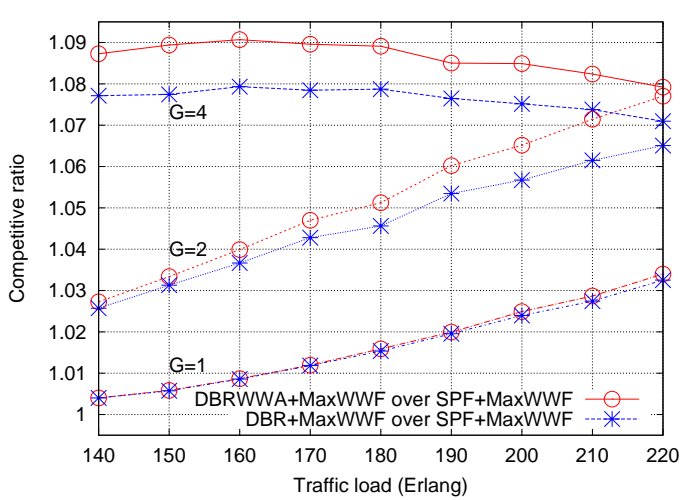

Figure 17. Competitive ratios of DBRWWA over SPF routing and of DBR over SPF routing with the MaxWWF algorithm

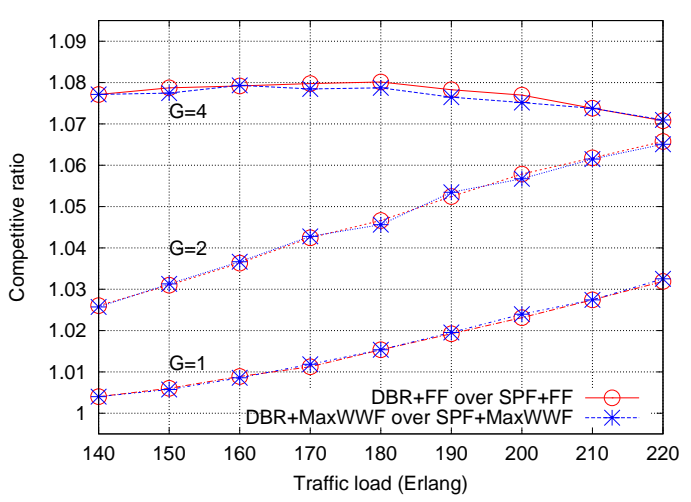

Figure 18. MaxWWF vs. first-fit with DBR for the routing subproblem

show that the competitive ratios of DBR/DBRWWA are approximately the same for first-fit and MaxWWF algorithms.

\section{CONCLUSIONS}

We extend DBR, which was originally proposed for opaque networks, to be applicable for transparent optical networks with WBS. To construct the proposed scheme called DBRWWA, we formulate an ILP problem for an MG-OXC-based network with the objective to minimize the total link cost 


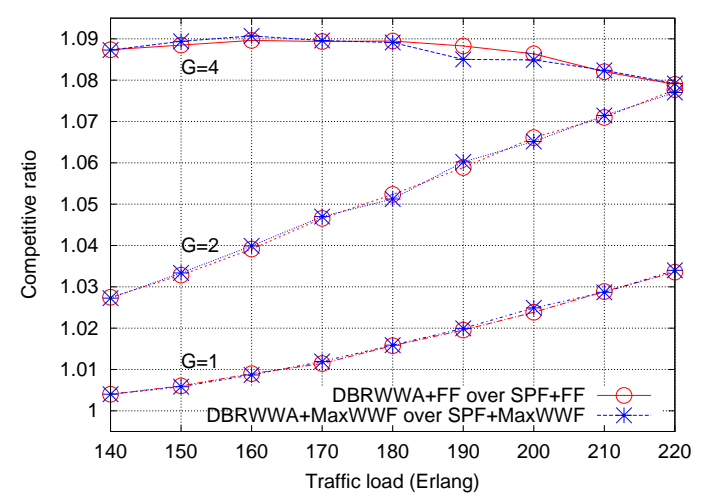

Figure 19. MaxWWF vs. first-fit with DBRWWA for the routing subproblem

subject to the constraints on traffic demands and the number of available switch ports at each node. Similar to DBR, DBRWWA guides online routing of dynamic lightpath requests, where optimized paths are computed offline. Compared to the commonly used SPF routing which does not take into account traffic statistics or the current network state, DBR and DBRWWA yield lower blocking probabilities. In addition, since DBRWWA considers waveband and wavelength assignment as well as the switch port limitations at network nodes, it provides an improvement on the blocking probability and the throughput compared to DBR.

For waveband and wavelength assignment that follow the routing process, we investigated two novel heuristics called MaxWWF and MinWWF to be investigated along with the existing first-fit and random-fit algorithms. Simulation results indicate that the first-fit and MaxWWF algorithms yield approximately the same blocking probabilities which are lower than those obtained from the other algorithms.

\section{ACKNOWLEDGMENT}

The first author would like to thank University of Engineering and Technology, Peshawar, Pakistan, for providing financial supports for doctoral study in Telecommunications field of study at the School of Engineering and Technology, Asian Institute of Technology, Thailand.

\section{REFERENCES}

[1] S. Bigo et al., "1.5 Terabit/s WDM transmission of 150 channels at $10 \mathrm{Gbit} / \mathrm{s}$ over $4 \times 100 \mathrm{~km}$ of TeraLight fibre," in Proc. Euro. Conf. Optical Commun. (ECOC), France, pp. 40-41, 26-30 Sept. 1999.

[2] L. Noirie, M. Vigoureux, and E. Dotaro, "Impact of intermediate traffic grouping on the dimensioning of multi-granularity optical networks," in Proc. Optical Fiber Commun. (OFC) Conf. and Exhibit, USA, vol. 2, 17-22 Mar. 2001.

[3] K. Harada, K. Shimizu, T. Kudou, and T. Ozeki, "Hierarchical optical path cross-connect systems for large scale WDM networks," in Proc. Optical Fiber Commun. (OFC) Conf. and Exhibit, USA, vol. 2, pp. 356-358, 21-26 Feb. 1999.

[4] P.-H. Ho and H.T. Mouftah, "Routing and wavelength assignment with multigranularity traffic in optical networks," $J$. Lightwave Tech., vol. 20, no. 8, pp. 1292-1303, Aug. 2002.
[5] M. Lee, J. Yu, Y. Kim, C.-H. Kang, and J. Park, "Design of hierarchical crossconnect WDM networks employing a twostage multiplexing scheme of waveband and wavelength," IEEE J. Sel. Areas Commun., vol. 20, no. 1, pp. 166-171, Jan. 2002.

[6] X. Cao, V. Anand, and Q. Qiao, "A waveband switching architecture and algorithm for dynamic traffic," IEEE Commun. Letters, vol.7, no.8, pp. 397-399, Aug. 2003.

[7] P.-H. Ho, H. Mouftah, and J. Wu, "A scalable design of multigranularity optical cross-connects for the next-generation optical Internet," IEEE J. Sel. Areas Commun., vol. 21, no. 7, pp. 1133-1142, Sept. 2003.

[8] X.-J. Kuo and C. Chen, "A blocking probability model in multigranularity cross-connect optical networks," in Proc. Workshop on High Performance Switching and Routing (HPSR), USA, 30 May-1 Jun. 2007.

[9] X. Cao, Q. Qiao, V. Anand, and J. Li, "Wavelength assignment in waveband switching networks with wavelength conversion," in Proc. IEEE GLOBECOM, USA, vol.3, pp. 1943-1947, 29 Nov.-3 Dec. 2004.

[10] X. Cao, V. Anand, and J. Li, and C. Xin, "Waveband switching networks with limited wavelength conversion," IEEE Commun. Letters, vol. 9, no. 7, pp. 646-648, Jul. 2005.

[11] X. Cao, V. Anand, and Q. Qiao, "Waveband switching for dynamic traffic demands in multigranular optical networks," IEEE/ACM Trans. on Netw., vol. 15, no. 4, pp. 957-968, Aug. 2007.

[12] M. Li, W. Yao, and B. Ramamurthy, "Same-destinationintermediate grouping vs. end-to-end grouping for waveband switching in WDM mesh networks," in Proc. IEEE ICC, Korea, vol. 3, pp. 1807-1812, 16-20 May 2005.

[13] M. Li and B. Ramamurthy, "Integrated Intermediate Waveband and Wavelength Switching for Optical WDM Mesh Networks," in Proc. IEEE INFOCOM, Spain, 23-29 Apr. 2006.

[14] H. Zang, J. Jue, and B. Mukherjee, "A review of routing and wavelength assignment approaches for wavelength routed optical WDM networks," Opt. Networks Magazine, vol. 1, pp. 47-60, Jan. 2000.

[15] J. Simmons, Optical Network Design and Planning (B. Mukherjee, ED.), Springer, 2008.

[16] R. Ramaswami and K.N. Sivarajan, Optical Networks: A Practical Perspective, 2nd Ed., Morgan Kaufmann, 2002.

[17] I. Chlamtac, A. Ganz, and G. Karmi, "Lightpath communication: An approach to high bandwidth optical WANs," IEEE Trans. on Commun., vol. 40, no. 7, pp. 1171-1182, Jul. 1992.

[18] A. Elwalid, D. Mitra, I. Saniee, and I. Widjaja, "Routing and protection in GMPLS networks: From shortest paths to optimized designs," J. Lightwave Tech, vol. 21, no. 11, pp. 2828-2838, Nov. 2003.

[19] C. Chen, X.-J. Kuo, Y.-Y. Chen, and T.Y. Lo, "A new model for optimal routing and wavelength assignment with fixedlength tunnel allocation in multigranularity cross-connect WDM networks," in Proc. IEEE MILCOM, USA, pp. 1270-1276, 17-20 Oct. 2005.

[20] M. Kovacevic and A. Acampora, "Benefits of wavelength translation in all optical clear channel networks," IEEE J. Sel. Areas Commun., vol. 14, no. 5, pp. 868-880, Jun. 1996.

[21] A. Sridharan and K.N. Sivarajan, "Blocking in all-optical networks," IEEE/ACM Trans. on Netw., vol. 12, no. 2, pp. 384-397, Apr. 2004.

[22] T. Stern and K. Bala, Multiwavelength Optical Networks: A Layered Approach, Prentice Hall, 1999.

[23] R. Randhawa and J. Sohal, "Static and dynamic routing and wavelength assignment algorithms for future transparent networks," Int. J. for Light and Electron Optics, vol. 121, no. 8, pp. 702-710, Apr. 2010.

[24] Python, http://www.python.org/.

[25] L.G. Tan and T. Sinclair, "Wavelength assignment between the central nodes of the COST 239 European Optical Network," 
in Proc. UK Performance Engineering Workshop on the COST 239 EON Proc., pp. 235-247, 1995.

[26] S. Plotkin, "Competitive routing of virtual circuits in ATM networks," IEEE J. Sel. Areas Commun., vol. 13, no. 6, pp. 1128-1136, Aug. 1995.

Akhtar Nawaz Khan received the B.Sc. (Hons) degree in Electrical Engineering from the University of Engineering and Technology, Peshawar, Pakistan, in 2006, and the M.Eng. degree in Telecommunications from the Asian Institute of Technology, Thailand, in 2009. $\mathrm{He}$ is currently studying in the Asian Institute of Technology, Thailand, where he is working towards the D.Eng. degree. His research interests include optimization for communication networks and optical networks especially routing, waveband and wavelength assignment in WDM optical networks with wavelength switching as well as waveband switching.

Poompat Saengudomlert received the King Scholarship from Thailand in 1991 to earn the B.S.E. degree in electrical engineering from Princeton University, Princeton, NJ, in 1996. He then received the M.S. and Ph.D. degrees, both in electrical engineering and computer science, from Massachusetts Institute of Technology (MIT), Cambridge, in 1998 and 2002, respectively.

From 2002 to 2004, he was a Postdoctoral Associate at the Laboratory for Information and Decision Systems at MIT. From 2005 to 2011, he was an Assistant Professor at the Asian Institute of Technology (AIT), Thailand. Since July 2011, he has been an Associate Professor at AIT. His research interests include communication theory, optical communications, and network resource allocation problems. 